\title{
Cognitive reserve masks neurobehavioral expression of human immunodeficiency virus- associated neurological disorder in older patients
}

This article was published in the following Dove Press journal:

Neurobehavioral HIV Medicine

28 October 20II

Number of times this article has been viewed

\author{
April D Thames ${ }^{1,3}$ \\ Jessica M Foley ${ }^{2}$ \\ Stella E Panos 1,3 \\ Elyse J Singer' \\ Suzie El-Saden ${ }^{1,3}$ \\ Charles H Hinkin ${ }^{1,3}$ \\ 'University of California, Los Angeles \\ School of Medicine, Los Angeles, \\ CA; ${ }^{2}$ Boston VA Healthcare System/ \\ Harvard Medical School, Boston, MA; \\ ${ }^{3}$ VA Greater Los Angeles Healthcare \\ System, Los Angeles, CA, USA
}

Background: Cognitive reserve theory may help to explain why some individuals are better able to withstand greater neurological insult before neurobehavioral manifestations occur. The present study provides a direct test of the cognitive reserve hypothesis in a sample particularly predisposed to cognitive impairment, ie, older human immunodeficiency virus (HIV)-positive adults.

Methods: Eighteen older HIV-positive adults, of mean (standard deviation) age $53.25 \pm 4.2$ years, with $13.5 \pm 2.0$ years of education, underwent magnetic resonance imaging and neuropsychological assessment. Regions of interest in the basal ganglia were extracted from $\mathrm{T} 1$ weighted scans and volumetric measurements were acquired. Cognitive reserve capacity was measured using premorbid intelligence quotient estimates and years of education. Participants were matched based on global neuropsychological performance.

Results: Individuals with higher levels of cognitive reserve demonstrated greater atrophy in the basal ganglia compared with individuals with lower levels of reserve, despite consistent cognitive function.

Conclusion: Older HIV-positive patients with higher levels of cognitive reserve may appear cognitively healthier than their striatal integrity would suggest.

Keywords: cognitive reserve, human immunodeficiency virus, magnetic resonance imaging, aging, basal ganglia

\section{Introduction}

Human immunodeficiency virus (HIV) is in the class of retroviruses that disrupt functioning of the central nervous system. Despite advances in antiretroviral therapy, approximately $30 \%-60 \%$ of patients will report cognitive decline during the course of their illness. ${ }^{1,2}$ HIV preferentially targets subcortical white and gray matter, particularly the basal ganglia, although other brain regions are also affected (eg, the hippocampus). Studies using voxel-based morphometry have found atrophy of the basal ganglia in patients with $\mathrm{HIV}^{3,4}$ that results in dopaminergic abnormalities. ${ }^{5} \mathrm{~A}$ recent study demonstrated that atrophy of the basal ganglia persists despite implementation and maintenance of antiretroviral treatment. ${ }^{6}$

Nevertheless, a common conundrum facing clinicians working with HIV patients is why some patients demonstrate overt signs of disease whereas others do not, despite equivalent biomarkers of disease progression. Of particular interest is that, irrespective of HIV-related immunosuppression, some individuals afflicted with HIV fail to demonstrate HIV-associated neurocognitive dysfunction. ${ }^{7-9}$
Correspondence: April D Thames UCLA School of Medicine, Department of Psychiatry and Biobehavioral Sciences, 760 Westwood Plaza, Los Angeles, CA 90095 , USA

Tel + I 3I0 478 37II ext 49565

Fax + I 3102068525

Email athames@mednet.ucla.edu 
Cognitive reserve capacity, which refers to the threshold of brain damage that must be sustained before an individual will display overt neurobehavioral impairment, ${ }^{10,11}$ may help to explain neurobehavioral differences observed in HIV and other neurodegenerative diseases. The effects of cognitive reserve may particularly explain the potential for preserved (or greater than expected) cognitive function in some individuals despite the presence of such neuropathology. ${ }^{12-17}$

Cognitive reserve was first introduced as a protective factor that preserved cognitive skills in the face of pathological effects associated with normal aging. ${ }^{13,15,17}$ The study of cognitive reserve has also been applied to neurodegenerative disorders of aging, ${ }^{14,18}$ particularly Alzheimer's disease. Within the aging and dementia literature, two models have been proposed to explain neural compensation. The first is the "brain reserve capacity" model. The brain reserve capacity model focuses on anatomical correlates, suggesting that passive factors (such as the number of synapses or brain volume) confer a particular capacity to endure neuropathological processes. The brain reserve capacity model serves to mask neurobehavioral manifestations until a critical threshold is reached.

A major criticism of the passive brain reserve capacity model is that it does not account for individual differences in cognitive or functional processing. To address this concern, a "functional" model of reserve, ie, the cognitive reserve hypothesis, was conceptualized..$^{19}$ In this hypothesis, cognitive reserve reflects the ability to use cognitive processes and brain networks in an effective way. Neural compensation, on the other hand, refers to adopting new compensatory brain networks after pathology has impacted those networks typically utilized for particular tasks. Neural compensation, and accordingly, the cognitive reserve hypothesis, emphasizes the ability to withstand age-related processes or Alzheimer's disease pathology. ${ }^{20}$ Studies of cognitive reserve in aging suggest that individuals with higher cognitive reserve may be better able to compensate for cognitive deficits resulting from neurodegeneration.

Applying the cognitive reserve model in aging/dementia to HIV has provided critical insights into factors that protect against the development of HIV-associated cognitive dysfunction. It has been demonstrated that educational attainment ${ }^{21}$ and estimated premorbid intelligence quotient (IQ) ${ }^{7}$ tend to buffer the deleterious effects of HIV neuropathology. Hence, active cognitive reserve factors, such as educational attainment, estimated premorbid intellectual function, and occupational complexity, may increase the threshold for neuropsychological dysfunction in the presence of cognitive risk factors, such as advancing age and neurological disease..$^{13-18}$
Using positron emission tomography, Stern et al conducted a series of seminal studies demonstrating this phenomenon in patients with Alzheimer's disease..$^{22}$ After matching subjects on Mini-Mental State Examination scores, they found that patients with higher levels of cognitive reserve demonstrated a greater degree of cerebrometabolic reduction in the parietotemporal region. Among HIV-seropositive participants with low levels of cognitive reserve, deficits in frontal-striatal functions, such as information processing speed, attention, verbal learning and memory, and executive functioning, have been documented..$^{23}$

There is reason to expect that older adults with HIV may be at greater risk for cognitive decline. Studies have found that the longer an individual is infected with HIV, the greater the loss in subcortical volume. ${ }^{6}$ Furthermore, there is an increased rate of HIV-associated dementia among older patients..$^{24,25}$ The Multicenter AIDS Cohort Study identified a relative hazard ratio for dementia of 1.60 per decade of life at AIDS onset. ${ }^{26}$ Similarly, after controlling for duration of infection, use of highly active antiretroviral therapy, and CD4 T lymphocyte count, older patients are three times more likely to meet criteria for HIV-associated dementia in a research setting. ${ }^{25}$ Whether there is an additive or synergistic relationship between aging and HIV on neuropsychological testing performance is not fully known, but there is compelling evidence that HIV accelerates the brain aging process. ${ }^{27}$

Therefore, it is plausible that cognitive reserve conceals the expression of underlying differences in HIV neurodegeneration. The purpose of the current study was to determine whether cognitive reserve functions in a similar manner in HIV when compared with other dementias, including Alzheimer's disease, with notable functional discrepancies between underlying neuropathology and cognitive outcomes based upon cognitive reserve status. Although prior work has documented the effects of cognitive reserve upon cognition alone, what remains to be investigated is what role, if any, cognitive reserve status plays in the integrity of striatal function among older adults with HIV. The current investigation focused on older adults, a group of scientific and clinical relevance given that older adults are one of the fastest growing (and to date largely understudied) segments of the HIV-infected population.

To test this hypothesis, we used an approach similar to that used by Stern et al, ${ }^{22}$ whereby magnetic resonance imaging was used to determine whether neurocognitively matched HIV-infected patients with higher levels of cognitive reserve would demonstrate greater brain atrophy than patients with lower levels of cognitive reserve. Cognitive reserve theory would predict that patients with higher levels 
of cognitive reserve could withstand a greater degree of underlying neurological damage before this would be evident in neuropsychological function. Accordingly, given the predilection of HIV for basal ganglia pathology, we anticipated that, despite equivalent extent of overt neurocognitive compromise, patients with higher levels of cognitive reserve would demonstrate a greater degree of striatal atrophy.

\section{Materials and methods}

Participants in this study were HIV-positive adults, aged 18 years and older (mean $53.25 \pm 4.2$ years), with a mean length structures of interest. ${ }^{28}$ Basal ganglia were selected for study because HIV has been shown to damage the striatum preferentially (see Figure 1).

\section{Cognitive reserve capacity}

Participants were administered the Wechsler Test of Adult Reading $^{29}$ to assist in calculating premorbid IQ estimates. Cognitive reserve was estimated based upon a median split of a composite score which averaged the sum of premorbid IQ and standardized years of education. Standardized years of education was calculated based upon the formula:

$$
\text { Standardized education }=\left[\frac{\text { Years of education }- \text { average years of education }(\text { sample })}{\text { Standard deviation of the sample }}\right] \times 15+100
$$

of education of $13.5 \pm 2.0$ years, recruited from local infectious disease clinics and community agencies as part of a larger study examining the effects of aging and HIV on neuropsychological outcomes. Exclusion criteria were inability to provide informed consent, a neurological disorder, such as HIV-associated central nervous system opportunistic infection/neoplasm, moderate-severe head injury, seizure disorder, demyelinating illness, cerebrovascular accident, current psychotic spectrum disorder or mood disorder, substance abuse/dependence within the past year, or if magnetic resonance imaging was contraindicated (eg, due to claustrophobia or metallic inclusions).

\section{Acquisition of magnetic resonance images}

Structural magnetic resonance images were obtained using a 1.5 T Magnetom Sonata scanner (Siemens AG, Erlangen, Germany), using a single-shot, echo-planar acquisition sequence (repetition time/echo time 10,000/88 mS, four $\mathrm{b}=0 / \mathrm{b}=750 \mathrm{~S} / \mathrm{mm}^{2}$, four averages, matrix $128 \times 128$, field of view $256 \times 256 \mathrm{~mm}$, and slice thickness $2 \mathrm{~mm}$ ). The protocol included an initial multiplanar scout, an axial-oblique, proton density/T2 weighted double spin echo, and sagittal whole brain high-resolution $\mathrm{T} 1$ weighted magnetic resonance image sequences. Scan planes were oriented perpendicular to the anterior-posterior commissure line.

\section{Segmentation of region of interest}

Basal ganglia (caudate, putamen) regions of interest were extracted from the $\mathrm{T} 1$ weighted scans using the University of California, Los Angeles Laboratory of Neuroimaging BrainParser software, an automated learning-based algorithm that efficiently performs whole brain image segmentation to parse an input magnetic resonance image into 56 anatomical
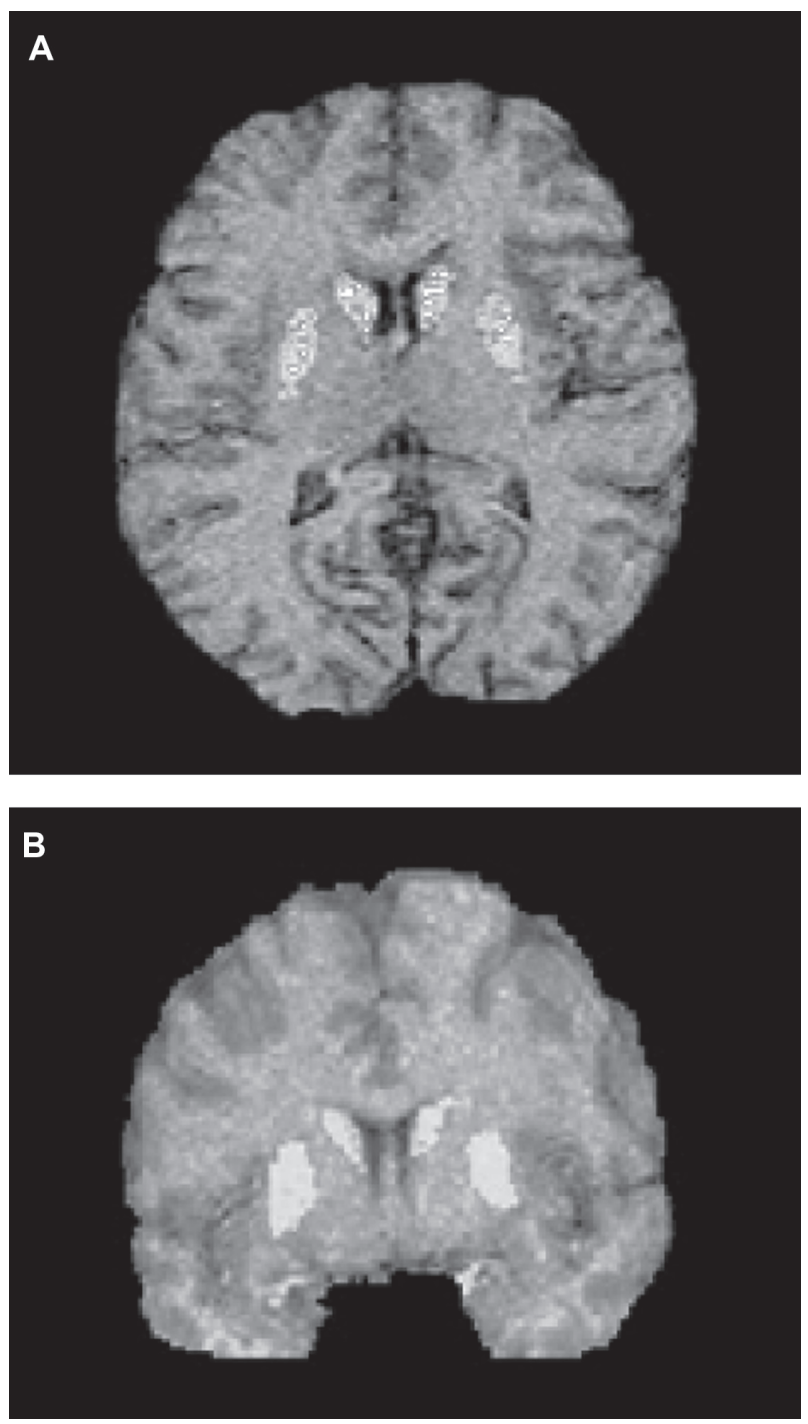

Figure I Basal ganglia regions of interest (caudate and putamen extractions) (A) axial view, (B) coronal view. 
A median split of the cognitive reserve score was used to classify individuals as "low" and "high" reserve.

\section{Neurocognitive assessment}

All participants completed a comprehensive neuropsychological battery, for which a description of testing and normative data has been published elsewhere, ${ }^{30}$ administered by trained psychometrists supervised by a board-certified neuropsychologist $(\mathrm{CHH})$. Test scores were converted to demographically (age, ethnicity, and education) corrected $\mathrm{T}$ scores (mean $50 \pm 10$ ) using published normative data, which yielded a global index of neuropsychological function against which cognitive reserve groups were matched at an individual participant level.

\section{Data analysis and procedures}

Caudate and putamen values were first corrected for whole brain volume. Distributions of volumetric data and neurocognitive scores were inspected for normality and linearity. To examine the effects of cognitive reserve, participants were matched on global neurocognitive performance. Matchedgroups $t$-testing was used to examine differences in basal ganglia volume as a function of cognitive reserve capacity.

\section{Results}

\section{Demographics and primary study variables}

There were no significant differences between the cognitive reserve groups for demographic-related and disease-related variables such as age, past substance abuse, length of HIV infection, recent and nadir CD4 count, and viral load. Significant group differences were observed in ethnicity, years of education, and premorbid IQ (see Table 1). While HIV-related and past substance abuse variables failed to reach statistical significance, they yielded modest effect sizes. Therefore, a series of bivariate correlations and $t$-tests were conducted to determine if relationships existed between ethnicity, recent and nadir CD4 count, viral load, past substance abuse, and basal ganglia volume.

There were no statistically significant $(p>0.05)$ relationships between recent and nadir CD4 count and basal ganglia volume ( $r=0.08$ and $r=0.21$, respectively). Length of HIV infection was not significantly correlated with basal ganglia volume $(r=-0.09, p=0.89)$. There were no statistically significant basal ganglia volume differences between viral load (detectable [ $>50$ copies $/ \mathrm{mL}$ ] vs undetectable), $t(16)=-0.65, p=0.75)$ and substance abuse/dependence groups $[t(16)=-0.80, p=0.59]$. There were also no significant ethnic group differences for basal ganglia volume $[\mathrm{F}(2,15)=2.34, p=0.10]$.

\section{Cognitive reserve and basal ganglia volume}

Consistent with expectations based on cognitive reserve theory, participants with higher cognitive reserve demonstrated greater atrophy of the basal ganglia $[t(8)=3.63, p=0.007$, $d=1.47]$ despite similar levels of global neurocognitive function. More detailed analyses revealed greater bilateral

Table I Demographic characteristics of cognitive reserve groups

\begin{tabular}{|c|c|c|c|c|}
\hline & Low reserve $(n=9)$ & High reserve $(n=9)$ & Results & Effect size (d) \\
\hline Age in years & $54.3(3.2)$ & $55.1(5.3)$ & $p>0.05$ & 0.18 \\
\hline Ethnicity & & & $p<0.001$ & 2.35 \\
\hline$\%$ African American & $100 \%$ & $33 \%$ & & - \\
\hline$\%$ Caucasian & 0 & $44 \%$ & & - \\
\hline \% Hispanic & 0 & $23 \%$ & & - \\
\hline Years of education & $12.5(1.1)$ & I4.I (I.5) & $*_{p}=0.04$ & 1.21 \\
\hline Male \% & $63 \%$ & $75 \%$ & $p>0.05$ & 0.72 \\
\hline Median recent CD4 count & 331.0 (IQ range I65) & 434.5 (IQ 396) & $p>0.05$ & 0.72 \\
\hline Nadir CD4 count & 66 (IQ range I20) & I35 (IQ 245) & $p>0.05$ & 0.35 \\
\hline$\%$ Undetectable viral load & $62 \%$ & $50 \%$ & $p>0.05$ & - \\
\hline$\%$ AIDS diagnosis ${ }^{\mathrm{a}}$ & $66 \%$ & $77 \%$ & $p>0.05$ & 0.49 \\
\hline$\%$ on ARV medication & $100 \%$ & $100 \%$ & $p>0.05$ & - \\
\hline Length of HIV infection (years) & $16(1.2)$ & $17(2.4)$ & $p>0.05$ & 0.63 \\
\hline$\%$ Reported past abuse/dependence & $77 \%$ & $55 \%$ & $p>0.05$ & 0.88 \\
\hline Premorbid IQ & $86.3(19.4)$ & $113.0(13.7)$ & $*_{p}<0.01$ & $\begin{array}{l}\text { I.58 range }(42-107) \\
\text { range }(86-123)\end{array}$ \\
\hline Cognitive reserve score & $184.0(13.2)$ & $233.4(18.1)$ & $* p<0.001$ & 3.11 \\
\hline
\end{tabular}

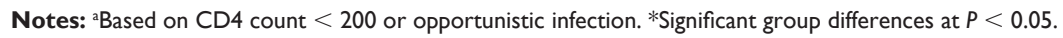

Abbreviations: AIDS, aqcuired immunodeficiency syndrome; ARV, antiretroviral; HIV, human immunodeficiency virus; IQ, intelligence quotient. 
caudate $[t(8)=2.33, p=0.04, d=0.79]$ and putamen $[t(8)=3.27, p=0.01, d=0.73]$ atrophy among individuals with higher cognitive reserve compared with individuals having lower levels of reserve (see Table 2).

\section{Discussion}

The present study provides a direct clinical test of the cognitive reserve hypothesis, and highlights an important factor to consider when treating patients with higher levels of premorbid intellectual function who present with neurodegenerative disorder. Consistent with this hypothesis, we found that after matching participants on neuropsychological performance, individuals with higher levels of cognitive reserve demonstrated greater atrophy of the basal ganglia. Our results suggest that older HIV-positive adults with higher levels of cognitive reserve may be able to shoulder greater disease burden before overt signs of disease emerge. Similar findings have been reported in studies of Alzheimer's disease, ${ }^{31,32}$ and also more recently for $\mathrm{HIV},{ }^{7,33}$ suggesting that these findings may be generalizable to a wider array of neurodegenerative disorders. Although already appreciated among other neurodegenerative conditions of aging, the findings of the present study also suggest that in the post-combination antiretroviral therapy era of HIV, cognitive reserve may apply to cognitive preservation in isolation, and that underlying neurobiological variables will remain predictably affected even in the presence of these protective mechanisms.

Investigation into the contribution of cognitive reserve for this vulnerable subgroup afflicted by the double assault of HIV-positive serostatus and older age has become an increasingly important objective, since some individuals with both risk factors fail to demonstrate significant neuropsychological deficits, and since little is understood about the reasons for this unexpected preservation of function. The results of the present study suggest that some individuals who remain at particular risk for cognitive compromise, and who present with documented evidence of neuropathological deterioration within areas known to be preferentially affected by the combined effects of advancing age and HIV infection, maintain adequate neuropsychological function, suggesting that cognitive reserve acts as a neuroprotective factor in preventing overt cognitive deterioration despite underlying physiological abnormality. Future studies, including longitudinal follow-up of individuals at similar risk with high cognitive reserve, should be conducted to determine whether cognitive deterioration proceeds more rapidly than would otherwise be expected after a threshold of neuropathological degeneration has been reached, as has been shown with a variety of other neurodegenerative populations, including Alzheimer's disease. Finally, prior work from our group ${ }^{34}$ has demonstrated that among a sample of subjects with varying levels of risk for cognitive dysfunction (HIV serostatus, age group), those at greatest risk but who maintain high cognitive reserve demonstrated preserved cognition at a level that exceeded all other groups, suggesting that cognitive reserve may successfully and preferentially protect individuals affected with HIV infection and advancing age. Therefore, future research should examine whether the relationship between varying risk for striatal neuropathological decline is similarly mediated by cognitive reserve status. Our findings, although preliminary, suggest that cognitive reserve delays the cognitive manifestations of underlying striatal pathology. Nevertheless, the concept of cognitive reserve in HIV would be further strengthened by replicating these results in other brain regions of interest (eg, frontal gray matter, hippocampus) that have also been demonstrated to atrophy as a result of HIV.

We recognize that our small sample size and convenience sampling methodology limits the generalizability of our findings to other subgroups, particularly for younger HIV cohorts. Furthermore, given the age restriction ( $\geq 50$ years) and the cross-sectional nature of the study, it is also possible that our sample may be undergoing normal age-associated neurological changes that could at least in part explain our current findings. While our study findings would be strengthened by inclusion of younger HIV-positive adults, identifying whether cognitive reserve may protect older HIV adults from cognitive decline was our primary study objective, because recent estimates suggest that over half of the individuals with HIV in the US will be over the age of 50 years by $2015 .{ }^{35}$

Table 2 Global neuropsychology and basal ganglia volumes

\begin{tabular}{lllll}
\hline & Low reserve $(\mathbf{n}=\mathbf{9})$ & High reserve $(\mathbf{n}=\mathbf{9})$ & Results & Effect size $(\mathbf{d})$ \\
\hline Global neuropsychology & $42.7(5.5)$ & $43.10(6.0)$ & $p>0.05$ & -0.06 \\
Basal ganglia & $7.3 \mathrm{~mm}^{3}(0.62)$ & $6.3 \mathrm{~mm}^{3}(0.73)$ & $* p=0.001$ & 1.47 \\
Bilateral caudate & $3.1 \mathrm{~mm}^{3}(0.74)$ & $2.5 \mathrm{~mm}^{3}(0.70)$ & $* p=0.04$ & 0.79 \\
Bilateral putamen & $4.1 \mathrm{~mm}^{3}(0.43)$ & $3.8 \mathrm{~mm}^{3}(0.38)$ & $* p=0.01$ & 0.73 \\
\hline
\end{tabular}

Note: *Statistically significant at $P<0.05$. 
Table 3 Individual participant values for global neuropsychology and basal ganglia volumes (reserve groups matched on global neuropsychological performance)

\begin{tabular}{|c|c|c|c|c|c|c|c|c|c|}
\hline $\begin{array}{l}\text { Low } \\
\text { reserve }\end{array}$ & Global NP* & $\begin{array}{l}\text { Basal } \\
\text { ganglia } \\
\text { volume }\end{array}$ & $\begin{array}{l}\text { Bilateral } \\
\text { caudate } \\
\text { volume }\end{array}$ & $\begin{array}{l}\text { Bilateral } \\
\text { putamen } \\
\text { volume }\end{array}$ & $\begin{array}{l}\text { High } \\
\text { reserve }\end{array}$ & Global NP* & $\begin{array}{l}\text { Basal } \\
\text { ganglia } \\
\text { volume }\end{array}$ & $\begin{array}{l}\text { Bilateral } \\
\text { caudate } \\
\text { volume }\end{array}$ & $\begin{array}{l}\text { Bilateral } \\
\text { putamen } \\
\text { volume }\end{array}$ \\
\hline 1 & 36.29 & $7.8 \mathrm{~mm}^{3}$ & $3.3 \mathrm{~mm}^{3}$ & $4.5 \mathrm{~mm}^{3}$ & I & 36.13 & $5.4 \mathrm{~mm}^{3}$ & $1.4 \mathrm{~mm}^{3}$ & $4.0 \mathrm{~mm}^{3}$ \\
\hline 2 & 37.05 & $6.5 \mathrm{~mm}^{3}$ & $2.7 \mathrm{~mm}^{3}$ & $3.8 \mathrm{~mm}^{3}$ & 2 & 37.14 & $5.2 \mathrm{~mm}^{3}$ & $1.8 \mathrm{~mm}^{3}$ & $3.4 \mathrm{~mm}^{3}$ \\
\hline 3 & 37.93 & $7.2 \mathrm{~mm}^{3}$ & $3.2 \mathrm{~mm}^{3}$ & $4.0 \mathrm{~mm}^{3}$ & 3 & 37.25 & $6.7 \mathrm{~mm}^{3}$ & $3.1 \mathrm{~mm}^{3}$ & $3.6 \mathrm{~mm}^{3}$ \\
\hline 4 & 39.97 & $7.5 \mathrm{~mm}^{3}$ & $4.9 \mathrm{~mm}^{3}$ & $3.6 \mathrm{~mm}^{3}$ & 4 & 40.47 & $6.7 \mathrm{~mm}^{3}$ & $3.1 \mathrm{~mm}^{3}$ & $3.6 \mathrm{~mm}^{3}$ \\
\hline 5 & 42.89 & $6.6 \mathrm{~mm}^{3}$ & $2.6 \mathrm{~mm}^{3}$ & $4.0 \mathrm{~mm}^{3}$ & 5 & 43.12 & $5.5 \mathrm{~mm}^{3}$ & $1.8 \mathrm{~mm}^{3}$ & $3.8 \mathrm{~mm}^{3}$ \\
\hline 6 & 44.88 & $6.8 \mathrm{~mm}^{3}$ & $2.6 \mathrm{~mm}^{3}$ & $4.2 \mathrm{~mm}^{3}$ & 6 & 44.95 & $6.4 \mathrm{~mm}^{3}$ & $2.8 \mathrm{~mm}^{3}$ & $3.7 \mathrm{~mm}^{3}$ \\
\hline 7 & 45.16 & $7.2 \mathrm{~mm}^{3}$ & $2.9 \mathrm{~mm}^{3}$ & $4.4 \mathrm{~mm}^{3}$ & 7 & 46.75 & $6.9 \mathrm{~mm}^{3}$ & $2.4 \mathrm{~mm}^{3}$ & $4.6 \mathrm{~mm}^{3}$ \\
\hline 8 & 48.62 & $7.4 \mathrm{~mm}^{3}$ & $2.5 \mathrm{~mm}^{3}$ & $4.9 \mathrm{~mm}^{3}$ & 8 & 49.18 & $7.3 \mathrm{~mm}^{3}$ & $3.3 \mathrm{~mm}^{3}$ & $4.1 \mathrm{~mm}^{3}$ \\
\hline 9 & 52.13 & $8.5 \mathrm{~mm}^{3}$ & $5.8 \mathrm{~mm}^{3}$ & $3.6 \mathrm{~mm}^{3}$ & 9 & 53.75 & $6.4 \mathrm{~mm}^{3}$ & $3.1 \mathrm{~mm}^{3}$ & $3.4 \mathrm{~mm}^{3}$ \\
\hline
\end{tabular}

Note: *Global T score.

Abbreviation: NP, neuropsychological.

Establishing such "proof of concept" among this population encourages further investigation into the neuroprotective effects of cognitive reserve in a younger HIV cohort. Despite these limitations, the effect sizes related to cognitive reserve were moderate, which suggests that our findings may be able to be replicated in a larger sample. Clinicians treating older HIV-positive patients with higher levels of cognitive reserve should be aware that such patients may appear cognitively intact despite progression of underlying neurodegeneration and consider this phenomena when arriving at diagnostic or treatment decisions. Our findings, albeit preliminary, may explain discrepancies in findings between neuropsychological function and functional outcomes and HIV brain imaging. Also, patients at greatest risk for neuropsychological compromise (HIV positivity and aging) receive most benefit from cognitive reserve mechanisms. Therefore, it appears that cognitive reserve successfully and preferentially protects individuals affected by HIV infection and advanced age. Future research should focus on the means by which protective mechanisms of cognitive reserve may contribute to a higher threshold for neuropsychological dysfunction. It is commonly believed that proxies for cerebral reserve support maintenance of neuroplasticity in cognitive aging. Stimulating cognitive activities, such as education and complex occupational roles, may support these neuroplastic mechanisms.

Findings addressing the impact of cognitive reserve among predisposed individuals may contribute to our understanding of a possible mechanism by which some individuals who remain at particular risk for neuropsychological compromise (HIV-positive, aging) fail to demonstrate evidence of cognitive impairment. Further, results of this study may highlight a possible functional discrepancy between neuropathology and cognitive function that may be attributable to cognitive reserve, which would imply that these variables cannot be reliably used as proxies for one another and are modifiable based upon cognitive reserve status. This is particularly relevant in clinical practice, because a neurocognitive-neuropathological discrepancy based upon cognitive reserve status suggests that clinical concern should be raised in the presence of high cognitive reserve and neuropsychologically intact status. Clinicians working with such individuals should therefore consider obtaining neuroimaging to detect subthreshold neurodegeneration so as to help with diagnostic decision-making and treatment planning. Prior studies have offered support that greater cognitive reserve may in fact serve as a defense against the neuropsychological presentation of normal aging and the neuropathological effects of neurodegenerative diseases, and the results of the current study suggest that these findings may extend to the HIV-infected population as well.

\section{Acknowledgments}

This work was supported by a Veteran's Administration merit review to $\mathrm{CHH}$ and a UCLA Center for AIDS research grant to JF.

\section{Disclosure}

The authors report no conflicts of interest in this work.

\section{References}

1. Bloom FE, Rausch DM. HIV in the brain: pathology and neurobehavioral consequences. J Neurovirol. 1997;3:102-109.

2. Grant I. Neurocognitive disturbances in HIV. Int Rev Psychiatry. 2008;20:33-47.

3. Aylward EH, Henderer JD, McArthur JC, et al. Reduced basal ganglia volume in HIV-1-associated dementia: results from quantitative neuroimaging. Neurology. 1993;43:2099-2104. 
4. Küper M, Rabe K, Esser S, et al. Structural gray and white matter changes in patients in HIV. J Neurol. 2011;258:1066-1075.

5. Berger JR, Arendt G. HIV dementia: the role of the basal ganglia and dopaminergic systems. J Psychopharmacol. 2000;14:214-221.

6. Becker JT, Maruca V, Kingsley LA, et al. Factors affecting brain structure in men with HIV disease in the post-HAART era. Neuroradiology. 2011. [Epub ahead of print.]

7. Basso MR, Bornstein RA. Estimated premorbid intelligence mediates neurobehavioral change in individuals infected with HIV across 12 months. J Clin Exp Neuropsychol. 2000;22:208-218.

8. Heaton RK, Grant I, Butters N, et al. The HNRC 500 - neuropsychology of HIV infection at different disease stages. HIV Neurobehavioral Research Center. J Int Neuropsychol Soc. 1995;1:231-251.

9. Miller EN, Seines OA, McArthur JC, et al. Neuropsychological performance in HIV-1 infected homosexual men. Neurology. 1990;40:197-203.

10. Satz P. Brain reserve capacity on symptom onset after brain injury. A formulation and review of evidence for threshold theory. Neuropsychology. 1993;7:273-295.

11. Stern Y. Cognitive reserve and Alzheimer's disease. Alzheimer Dis Assoc Disord. 2006;20:S69-S74.

12. Christensen H, Anstey KJ, Parslow RA, et al. The brain reserve hypothesis, brain atrophy and aging. Gerontology. 2007;53:82-95.

13. Richards M, Deary IJ. A life course approach to cognitive reserve: a model for cognitive aging and development? Ann Neurol. 2005; $58: 617-622$

14. Roe CM, Xiong C, Miller P, Morris JC. Education and Alzheimer disease without dementia: support for the cognitive reserve hypothesis. Neurology. 2007;68:223-228.

15. Staff RT, Murray AD, Deary IJ, Whalley LJ. What provides cerebral reserve? Brain. 2004;127:1191-1199.

16. Vance DE, Struzick TC. Addressing risk factors of cognitive impairment in adults aging with HIV: a social work model. J Gerontol Soc Work. 2007;49:51-77.

17. Whalley LJ, Deary IJ, Appleton CL, Starr JM. Cognitive reserve and the neurobiology of cognitive aging. Ageing Res Rev. 2004;3:369-382.

18. Andel R, Vigen C, Mack WJ, et al. The effect of education and occupational complexity on rate of cognitive decline in Alzheimer's patients. $J$ Int Neuropsychol Soc. 2006;12:147-152.

19. Stern Y, Gurland B, Tatemichi TK, Tang MX, Wilder D, Mayeux R. Influence of education and occupation on the incidence of Alzheimer's disease. JAMA. 1994;271:1004-1010.

20. Stern Y. Cognitive reserve. Neuropsychologia. 2009;47:2015-2018.

21. Satz P, Morgenstern H, Miller EN, et al. Low education as a possible risk factor for cognitive abnormalities in HIV-1: Findings from the Multicenter AIDS cohort study (MACS). J Acquir Immune Defic Syndr. 1993;6:503-511.
22. Stern Y, Alexander GE, Prohovnik I, Mayeux R. Inverse relationship between education and parietotemporal perfusion deficit in Alzheimer's disease. Ann Neurol. 1992;32:371-375.

23. Stern R, Silva S, Chaisson N, Evans DL. Influence of cognitive reserve on neuropsychological functioning in asymptomatic human immunodeficiency virus-1 infection. Arch Neurol. 1996;53:148-153.

24. Chiesi A, Seeber A, Dally LG, Floridia M, Rezza G, Vella S. AIDS dementia complex in the Italian National AIDS Registry: temporal trends (1987-93) and differential incidence according to mode of transmission of HIV-1 infection. J Neurol Sci. 1996;144:107-113.

25. Valcour V, Shikuma C, Shiramizu, B, et al. Higher frequency of dementia in older HIV-1 individuals. Neurology. 2004;63:822-827.

26. McArthur J, Hoover D, Bacellar H, et al. Dementia in AIDS patients: incidence and risk factors. Multicenter AIDS Cohort Study. Neurology. 1993;43:2245-2252.

27. Ances B, Florin V, Yeh MJ, et al. HIV infection and aging independently affect brain function as measured by functional magnetic resonance imaging. J Infect Dis. 2010;201:336-340.

28. Tu Z, Narr KL, Dollar P, Dinov I, Thompson PM, Toga AW. Brain anatomical structure segmentation by hybrid discriminative/generative models. IEEE Trans Med Imaging. 2008;27:495-508.

29. Wechsler D. Wechsler Test of Adult Reading. New York: Psychological Corporation; 2001.

30. Thames AD, Kim MS, Becker BW, et al. Medication and finance management among HIV-infected adults: The impact of age and cognition. J Clin Exp Neuropsychol. 2010;6:1-10.

31. Geerlings MI, Deeg DJH, Schmand B, Lindeboom J, Jonker C. Increased risk of mortality in Alzheimer's disease patients with higher education? A replication study. Neurology. 1997;49:798-802.

32. Snowdon DA, Kemper SJ, Mortimer JA, Greiner LH, Wekstein DR, Markesbery WR. Linguistic ability in early life and cognitive function and Alzheimer's disease in later life. Findings from the Nun Study. JAMA. 1996;275(7):528-532.

33. Pereda M, Ayuso-Mateos JL, Gómez del Barrio A, et al. Factors associated with neuropsychological performance in HIV-seropositive subjects. Psychol Med. 2000;30:205-217.

34. Foley J, Ettenhofer M, Kim MS, Bedhin N, Castellon SA, Hinkin CH. Cognitive reserve as a protective factor in older HIV-positive patients at risk for cognitive decline. Appl Neuropsychol. In press.

35. Centers for Disease Control and Pevention. CDC HIV/AIDS Facts: HIV/AIDS among persons 50 and older. Available from: http://www. cdc.gov/hiv/topics/over50/resources/factsheets/over50.htm. Accessed October 17, 2011.
Neurobehavioral HIV Medicine

\section{Publish your work in this journal}

Neurobehavioral HIV Medicine is an international, peer-reviewed, open access journal focusing on advances in research in HIV AIDS, with specific reference to the neurological, psychiatric and behavioral consequences of the disease, concomitan infections and specific antiretroviral therapy. The manuscript

\section{Dovepress}

management system is completely online and includes a very quick and fair peer-review system, which is all easy to use Visit http://www.dovepress.com/testimonials.php to read real quotes from published authors. 